Les dictionnaires bilingues des maîtres ou professeurs auteurs de manuels pédagogiques

\title{
Nathanaël Duez auteur du Guidon de la langue italienne (1641) et du Dittionario Francese Italiano (1659-1660) : un maître de langues entre continuité et innovation
}

\section{Antonella Amatuzzi}

\section{OpenEdition \\ Journals}

\section{Édition électronique}

URL : https://journals.openedition.org/dhfles/3927

DOI : $10.4000 /$ dhfles.3927

ISSN : 2221-4038

\section{Éditeur}

Société Internationale pour l'Histoire du Français Langue Étrangère ou Seconde

Édition imprimée

Date de publication : 1 juin 2016

Pagination : 27-50

ISSN : 0992-7654

Référence électronique

Antonella Amatuzzi, « Nathanaël Duez auteur du Guidon de la langue italienne (1641) et du Dittionario Francese Italiano (1659-1660) : un maître de langues entre continuité et innovation », Documents pour I'histoire du français langue étrangère ou seconde [En ligne], 56 | 2016, mis en ligne le 04 septembre 2017, consulté le 31 mars 2023. URL : http://journals.openedition.org/dhfles/3927 ; DOI : https:// doi.org/10.4000/dhfles.3927

Ce document a été généré automatiquement le 31 mars 2023.

Tous droits réservés 


\title{
Nathanaël Duez auteur du Guidon de la langue italienne (1641) et du Dittionario Francese Italiano (1659-1660) : un maître de langues entre continuité et innovation
}

\author{
Antonella Amatuzzi
}

Nathanaël Duez, enseignant d'italien, de français et d'allemand, est l'auteur d'une nomenclature en quatre langues, de grammaires du français et de l'italien et de deux dictionnaires ${ }^{1}$ qui eurent tous un succès remarquable au XVII ${ }^{e}$ siècle.

Sa production concernant le domaine linguistique italien comprend Le Guidon de la langue italienne (1641) et le Dittionario italiano e francese $1659 / 60)^{2}$. Elle a été prise en examen essentiellement en marge de larges enquêtes de portée générale (Mormile 1993 : 38-40 ; 1989: 75-78 ; Mattarucco 2003:29-31, 39-41 et passim ; Murano 2013 : 48-53).

Nous nous intéresserons à ces deux ouvrages en les situant dans le cadre des activités des professeurs de langues opérant aux Pays-Bas et nous en donnerons une description. Nous concentrerons ensuite notre attention sur le Dittionario, en soulignant ses points de force. Ils se dégageront de manière précise de la lecture que nous mènerons parallèlement, en perspective comparative, des Recherches italiennes et françoises d'Antoine Oudin.

Notre étude est motivée par le constat que, même si des travaux récents ont reconstruit la genèse de la lexicographie franco-italienne (Lillo 2008 \& 2013), les rapports que les différents ouvrages et les différents auteurs entretiennent entre eux restent à investiguer dans les détails. Elle entend apporter une contribution dans cette direction de recherche. 


\section{Nathanael Duez, professeur de langues aux Pays-Bas}

Né en 1609 à Altwiller, village d'Alsace fondé par des huguenots exilés, Nathanaël était le fils de Samuel d'Huet, ministre de culte protestant, qui l'initia au latin dès son plus jeune âge. Adolescent, il séjourna pendant trois ans à Strasbourg, où il apprit l'allemand. Il voyagea ensuite en Allemagne, en Italie, en Angleterre et à Paris pour une douzaine d'années, en travaillant comme maître de langues. En 1639 il s'établit à Leyde, où résidait son frère Andries. Il y exerça la profession de magister linguae gallicae et italicae jusqu'à sa mort, survenue vers 1660 .

Duez compte donc parmi les nombreux enseignants issus du milieu des refugiés (Loonen 2000) qui gagnaient leur vie en mettant à profit leurs compétences langagières et participaient ainsi au développement de la vie culturelle de la République des Provinces-Unies ${ }^{3}$.

Ils bénéficiaient du contexte socio-historique qui voyait le français en plein essor dans toute l'Europe et en particulier aux Pays-Bas, où il était une langue de distinction religieuse, sociale et professionnelle, diffusée à des couches de plus en plus vastes de la population (Van Strien-Chardonneau \& Kok Escalle 2010).

Puisque leur rémunération pouvait varier en fonction de la loi du marché (nombre d'élèves, concurrence), ils trouvaient souvent un complément de revenu dans la publication d'ouvrages pédagogiques. Duez collabora avec les Elzevier, célèbre famille de typographes-imprimeurs, chez lesquels il commença à publier ses travaux destinés surtout à un public adulte et à un marché non seulement hollandais mais international. Ses élèves étaient principalement des expatriés allemands de famille aisée qui fuyaient les horreurs de la guerre de Trente Ans ou, pour l'italien, des bourgeois francophones qui commerçaient ou voyageaient en Italie, pays dont la langue n'avait pas perdu le prestige culturel et littéraire qu'il avait atteint à la Renaissance (Lo Cascio 1997) et dans lequel on assistait, d'ailleurs, à un vrai engouement pour le français (Dardi 1992).

\section{Le Guidon de la langue italienne}

La première édition du Guidon de la langue italienne date de 1641. Il dut avoir la faveur du public si l'on en juge au nombre de réimpressions (cf. bibliographie) et aux mots de l'éditeur de la dernière, celle de 1685, qui écrit dans son avis Il libraro al lettore:

Tra tutte le grammatiche che hò veduto, e vedo comparire alla giornata per la

Lingua Italiana, non ne hò veduta una che venga più gradita, sia dagl'Intelligenti, come dagli stranieri quanto quella di Natanaele Duez $(1685: 4)$.

Le Guidon est, comme beaucoup d'autres manuels de langue de l'époque, une compilation de textes de nature diverse. Il rassemble une grammaire de la langue italienne rédigée en français, trois dialogues, une comédie, des « compliments italiens » et des proverbes 4 . Ces matériaux didactiques ne sont pas tous du cru de Duez.

Les trois dialogues sont empruntés à Catherin Ledoux, qui les avait insérés dans sa Schola Italica (Dulcis, 1605) ${ }^{5}$. Présentés sur deux colonnes, avec le texte italien et la traduction française en regard, ils ont comme sujets: I Del salutare, levarsi, \& pigliar licenza, con alcune demande (I De salüer, se leuer, \& prendre congé, auec quelques demandes), II Del bere \& mangiare (II Du boire \& manger) et III Degli esercitii, dellí alloggiamento, e di andare al letto. (III Des exercices, du logement, \& d'aller coucher). Ils seront reproposés tels quels par 
Lodewijk Meyer ${ }^{6}$ dans sa grammaire intitulée Italiaansche Spraakkonst (Amsterdam : Abraham Wolfgang 1672; cf. De Boer 1996: 71; Vanvolsem 2000:99) où la version française est accompagnée d'une traduction en néerlandais.

La Comédie de la Moresse (titre italien La Mora) est une imitation italienne de L'Eunuque de Térence, dont l'auteur est Giovanni Battista Calderari (Vicence : Brunelli 1588) mais que Ledoux avait republiée (Dulcis 1599) et qui figure également dans la Schola Italica.

Les Complimenti Italiani sont un recueil de plusieurs phrases et formules utiles dans des situations communicatives de la vie quotidiennes ${ }^{7}$ dont la source serait (Bingen 1987 : 73) les Duelli di complimenti d'Angelo Gabrieli (Venise : Ghirardo Imberti, 1629).

La Ghirlanda di varii fioretti \& proverbi italiani porte la signature de l'Arsiccio Intronato, pseudonyme de Antonio Vignali ou Vignale (1500-1559), écrivain et poète de la Renaissance italienne. Elle est datée « Di Milano, del mese degli asini, 1600 », mais elle est antérieure puisqu'elle se trouvait déjà dans Alcune lettere piacevoli una dell'Arsiccio Intronato in proverbi, l'altre di M. Alessandro Marzi, Cirloso Intronato (Sienne : Bonetti 1587) et à la suite de L'Eunuchus de Ledoux (mais non pas dans la Schola).

La grammaire proprement dite (84 pages dans l'édition de 1659) est divisée en onze parties, selon une organisation qui suit un schéma traditionnel: 1 De la Lecture et Prononciation, 2 Des Articles \& Declinaisons, 3 Des Degrez de Comparaison, 4 Du mouvement des noms, 5 De l'Apostrophe, 6 De l'Accent, 7 Des Pronoms, 8 Des Verbes \& Coniugaisons. 9 Des Aduerbes \& Interjections, 10 Des Conjontions, 11 Des Prepositions ${ }^{8}$.

La description des parties du discours est « encore fortement inspirée par la tradition grammaticale latine, ce qui se reflète, par exemple, dans le traitement de l'adjectif comme sous-classe du nom ou dans le maintien du système casuel pour la déclinaison des articles, des noms et des pronoms, ou encore, dans la conservation du mode optatif grec » (Szoc 2009 : par. 26, à qui nous renvoyons pour une analyse plus circonstanciée).

Il s'agit d'une grammaire basée sur une approche contrastive. Pour exposer les règles de la langue italienne l'auteur a souvent recours à la comparaison avec le français, comme dans les cas suivants :

C devant e, e, se prononce comme feroit tch en François, ou tsch en Allemand $(1659: 2)$.

Les Italiens ont aussi cinq sortes de pronoms, comme les François: assavoir personnels, possessifs, demonstratifs, interrogatifs \& relatifs $(1659: 23)$.

En évaluant l'ensemble des matériaux sélectionnés dans le Guidon, ainsi que le paratexte, nous pouvons estimer quelle est l'orientation pédagogique de Duez.

Dans sa préface, adressée Aux Amateurs de la Langue Toscane, il file la métaphore, que le titre du manuel annonçait, de l'apprentissage linguistique comme voyage, pouvant réserver des obstacles et des plaisirs.

Il articule sa réflexion en trois points. Premièrement il affirme :

Pour le commencement de ce voyage il y a deux chemins, l'vn peu long, comprenant tout pour les plus curieux, qui veulent tout savoir et l'autre assez court, ne contenant que les choses plus necessaires, marquées de grosses lettres. Et cettuy-cy sera pour ceux qui ne prennent point de plaisir à s'amuser à tant de menues obseruations (1659:3-4).

Dans le Guidon un parcours d'apprentissage personnalisé, qui répond aux exigences et aux motivations des élèves, est donc conçu et rendu possible par l'emploi de caractères typographiques plus grands pour les règles de base et plus petits pour les exemples, les annotations, les exceptions. 
Puis il poursuit, en écrivant :

Pour ce qui est des Dialogues, vous sçaurez, que plusieurs simples paroles en ont esté à dessein retranchées, d'autant que l'on en peut auoir suffisance dans la Porte des Langues du sieur Comenius, en nostre Vocabulaire, ou en quelque autre, selon que chacun le trouuera plus à son goust $(1659: 4)^{9}$.

La référence à Comenius, un des repères théoriques de Duez, qui avait fourni la première traduction en italien de la Janua aurea reserata (Duez 1640) est significative. Loonen (1994) en minimise l'influence : le caractère plus déductif et explicite dont fait preuve la démarche de Duez, chez qui le rôle de la grammaire serait privilégié, dépendrait du fait qu'il visait un public adulte et universitaire tandis que Comenius s'adressait à de jeunes écoliers. Or, le programme didactique de Duez, tel qu'il ressort du Guidon, a bien des aspects en commun avec l'approche suivie par Comenius et accentue le côté culturel et pragmatique de l'apprentissage.

Il met en effet bien en évidence dans son troisième point que la Comédie et la Ghirlanda servent à :

expliquer le vray sens de plusieurs façons de parler, particulieres aux Italiens comme ainsi soit que chasque langue en a quelques singulieres; ny plus ny moins que chasque nation a ses façons, manieres \& coustumes \& chasque pays ses singulieres proprietez, toutes diuerses \& differentes de celles des autres (1659:5).

Sa méthode conjugue théorie et pratique, finalités descriptives et pédagogiques. Il insiste sur les règles grammaticales et sur la mémorisation du vocabulaire mais il sait, grâce à sa propre expérience, que ses étudiants ont aussi besoin d'un contact direct avec la langue-cible pour pouvoir affronter des situations de communication quotidienne et il prévoit pour eux des supports adaptés.

\section{Le Dittionario : éditions, sources et filiation}

La première édition du Dittionario paraît en 1659-60, la dernière est de 1678. Pendant près de vingt ans il a plusieurs réimpressions, quasiment identiques dans le contenu et dans la mise en page, chez des éditeurs variés, en Italie, en France et en Suisse ( $c f$. bibliographie ${ }^{10}$ ), ce qui atteste d'une bonne diffusion internationale, à un moment où la scène lexicographique franco-italienne est dominée par les Recherches italiennes et françoises d'Antoine Oudin (sur lui cf. Pfister 1989, Minerva 2007).

Cette œuvre phare, «le dictionnaire bilingue le plus représentatif des tendances lexicographiques de l'époque quant à l'abondance des lemmes retenus et à leur microstructure " (Minerva 2013 : 37), avait paru en 1640 et eut trois rééditions chez le même éditeur, Antoine de Sommaville, en 1643, 1653 et 1655. En 1663 Lorenzo Ferretti, romain, secrétaire interprète et maître de langues à la Cour de Paris, en donne une nouvelle édition avec le titre Dictionnaire italien et françois. Contenant les recherches de tous les mots italiens expliquez en françois.

Il ne fait pas de doute que les Recherches sont la source primaire du Dittionario. Toutefois, alors que des lexicographes successifs, tels Ferretti ou Veneroni (Minerva, 1991 et 2013), se situent expressément dans la continuité d'Oudin, Duez semble vouloir s'en démarquer. Il n'avoue jamais sa dette, pourtant indéniable, envers son prédécesseur. Il se limite à affirmer dans la Préface du Dittionario :

[...] car bien qu'il y ait bon nombre de dictionnaires italiens dont les meilleurs qui ayent jamais esté sont celuy de Crusca, d'Oudin et de Françosin ${ }^{11}$, si est ce qu'il n'y 
en a point auquel il ne se trouve encor beaucoup de manquemens et d'imperfections.

Il décide de se lancer dans l'entreprise de rectifier les outils lexicographiques existants grâce à ses compétences et à son expérience, qu'il met en avant :

Car faisant profession de la langue françoise et italienne il y a plus de vingt quatre ans et le bon Dieu m'ayant fait la grace de me donner un assez bon talent en cette vacation, j'ose bien prendre la liberté de te dire (ce que soit dit sans vanterie) que je m'en suis acquis une assez bonne connoissance. Et ayant une bonne douzaine d'années commencé à remarquer plusieurs fautes et manquemens en divers Dictionnaires, qui auoient esté mis en lumiere de ces deux belles langues et particulierement de l'Italienne, j'ay creu estre obligé de mettre en fin telles remarques et corrections en bon ordre en quelque bonne edition et de les donner au public sur tout pour le proffit et auantage des jeunes gens qui voudront apprendre l'vne ou l'autre de ces deux excellentes langues et ce affin de leur y donner une plus claire et plus veritable explication des choses plus difficiles et obscures que peut-estre ils n'en trouveront pas en d'autres (Préface).

Duez ne modifie pas foncièrement la nature et l'organisation des Recherches et, bien qu'il le présente comme profitable pour apprendre les deux langues, le Dittionario reste un ouvrage s'adressant majoritairement à des utilisateurs francophones.

Son exercice de remaniement est tout de même loin d'être négligeable. En tant qu'enseignant, l'intelligibilité et l'exhaustivité étaient pour lui des objectifs prioritaires. En effet, comme l'illustreront les exemples qui seront présentés plus loin, les changements qu'il introduit vont dans la direction d'une simplification de la structure des articles d'une part et d'un enrichissement des contenus de l'autre.

Le travail accompli par Duez peut être apprécié à sa juste mesure lorsqu'on examine les relations intertextuelles existantes entre le Dittionario, les Recherches et le Dictionnaire Italien et François de Laurent Ferretti.

C'est pour cette raison que nous avons procédé à des vérifications ponctuelles desquelles il résulte que l'intervention auctoriale de Duez est bien visible : il effectue une révision critique des Recherches, amplifie et améliore. En revanche Ferretti se contente de republier le matériel lexicographique d'Oudin avec quelques additions (touchant principalement la prononciation), sans le transformer et, étonnamment, sans tenir compte des ajustements et compléments de Duez.

Nous nous situons donc dans le sillage de l'analyse linguistique comparée des Recherches et de leur progéniture effectuée par Minerva (2007) ${ }^{12}$, pour constater que le Dittionario reste un ouvrage unique et isolé, qui n'a pas eu de postérité.

\section{Le Dittionario : les nouveautés}

La nomenclature du Dittionario est essentiellement celle des Recherches, comprenant un nombre limité de noms propres et de toponymes (pour la plupart entrant dans des phraséologismes) ${ }^{13}$, des archaïsmes, des régionalismes, des mots scientifiques et techniques (surtout concernant les champs lexicaux de la marine, de la botanique, de la médecine et de la zoologie).

Comme chez Oudin, plusieurs registres de langues sont présents: des mots bas et familiers côtoient des termes littéraires et rares.

La modification la plus évidente consiste dans l'élimination presque systématique des mots qu'Oudin avait indiqués comme relevant du jargon ${ }^{14}$. Dans sa Préface Duez se 
plaint en effet de certains auteurs de dictionnaires qui « en ont trop fait en y mettant des mots feints et inusités comme ceux du jargon et des nations qui ne parlent pas bon Italien ou François ».

Il tient au contraire à garder les "mots anciens » qu'il signale par un astérisque, comme avait déjà fait Oudin. Il avertit :

Pour ce qui est des mots marqués d'vne estoille, cela donne à entendre que ce sont des mots surannés qui ne sont plus en vsage, ou des mots trop latins ou des termes de quelque dialecte qui n'est pas bon françois ny bon italien et par ainsi telles paroles ne sont pas de bonne mise. Mais il est pourtant necessaire d'auoir les paroles anciennes dans le dictionnaire pour pouvoir entendre quelques bons vieux autheurs ésquels il s'en trouve souvent comme Iehan Villani, Crescentio, Boccace, Dante, Petrarque, Aretin, Guichardin, Machiavel, et d'autres (Préface).

On observe, de même que dans les Recherches, un manque de bidirectionnalité. Le Dittionario aborde l'italien en tant que langue cible, comme le prouve la longueur inégale des deux parties (970 pages pour l'italien-français, partie version, et 605 pages pour le français-italien, partie thème); il répond plus aux nécessités des locuteurs francophones qu'italophones et l'objectif est le décodage plus que l'encodage. Les deux nomenclatures ne sont pas parfaitement renversées : tous les équivalents donnés dans une partie ne figurent pas dans l'autre. Ainsi, la partie italien-français comporte l'entrée « MAGNANO : serrurier, forgeron, chaudronnier » mais ce mot vedette n'apparaît dans aucun des trois articles correspondant à ses traductions dans la partie françaisitalien, où l'on trouve :

SERRURIER : chiavaro

FORGERON : fabbro \& ferraro

CHAUDERONNIER : calderaio ò calderaro, paiuolaro \& conzalavezzi ${ }^{15}$

Au niveau de la structure, le Dittionario adopte un nouveau système de renvois expliqué dans la Préface:

Quand le lecteur est renuoyé d'vn mot à un autre par vn vedi ou un voyez cela signifie non seulement qu'il y trouvera la vraye explication de ce qu'il cherche mais aussi pour donner à entendre que la parole à laquelle il est renvoyé est meilleure et mieux ortographiée que l'autre.

Nous trouvons donc :

INGOSSARE, voyez ingozzare

INGOZZARE, engorger engloutir

ou :

TANESIE, voyez tanaisie

TANAISIE, atanasia ò tanaceto, herba

là où Oudin répétait deux fois la même définition sous deux entrées distinctes ou traitait les variantes graphiques sous une entrée unique, en rendant la consultation moins aisée.

Le plus grand mérite de Duez réside dans la réorganisation des articles. Il est le premier, dans la lexicographie franco-italienne, à numéroter les différentes acceptions des mots à la suite du mot-vedette :

(s.v. MELLONE)

Oudin: melon. Par similitudes, les fesses.

Duez : 1 melon 2 le derriere, les fesses 3 un niais et vn benest 
Les traductions des mots polysémiques, traités dans les dictionnaires de la série OudinVeneroni dans des articles autonomes, qui multipliaient les entrées, sont réunis dans un même paragraphe :

(s.v. PLACE)

Oudin : luogo

piazza, item mercato

Duez : I luogo 2 piazza 3 mercato 4 città ò fortezza

(s.v. VERSO)

Oudin : vers, composition poëtique

l'endroit de l'estoffe

endroit, situation, costé. La note d'vn oiseau

vers, envers

Duez : subst. I vn vers, composition poëtique 2 l'endroit ou le droit d'vne étoffe $3 \mathrm{Vn}$

endroit, vn costé 4 vne sorte, façon ou manière 5 la note ou le chant d'vn oiseau

prepos I vers, devuers, 2 enuers, à l'endroit de \&c.

Les homonymes et homographes sont soit dégroupés (par exemple pour vol, vase, nettare, foro il y a deux entrées) soit numérotés à la suite du mot-vedette :

(s.v. MINUTO)

Oudin : menu. Vne minute Et vne menesrte ou potage d'herbes ou poirées hachées

menu.

Item, vne sorte de monnoye

Duez: I menu 2 vne minute 3 vne menestre ou potage d'herbes ou porrée hachée

menüe 4 vne sorte de monnoye

(s.v. TASSO)

Oudin: if, arbre. Vne sorte d'enclue. Vn tesson, animal.

Duez : I if, vn arbre 2 vne sorte d'enclume 3 vn taisson ou blaireau, animal

L'activité de grammairien de Duez a produit des effets perceptibles sur celle de lexicographe. Il intègre dans le Dittionario des informations concernant les parties du discours, au moins lorsqu'elles sont indispensables pour faire des distinctions et dans le choix des traductions :

(s.v. MANCO)

Oudin : defectueux : gauche. Pour foible. \& manquement

moins

Duez : adv. I moins 2 pas mesmes, non plus, aussi peu, encor moins

adject. 1 defectueux. 2. gauche. 3. sinistre, malheureux 4. foible

subst. faute ou manquement

(s.v. GAGLIOFFA)

Oudin : una Gaglioffa, Vne saloppe, Vne gaupe Gaglioffa, mot lomb. Pochette, poche, sac, besace

Duez : adject. vne saloppe, vne gaupe, \& vne fripponne.

subst. vne pochette, poche, ou besace, en Lombardie.

La décision de mettre à la disposition du lecteur ces données que les Recherches omettaient est révélatrice d'un effort de pédagogisation puisqu'une réflexion sur la langue est favorisée.

Toujours dans l'intention de rendre son Dittionario plus efficace, Duez est plus scrupuleux qu'Oudin quand il fournit des notations relatives à la prononciation, réservées à la partie italien-français et aux cas se prêtant à confusions et erreurs. Pour la transcription phonétique il se sert des lettres de l'alphabet :

(s.v. MEZzo)

Oudin : prononcé rude, passé, sec, flestry

demy : milieu : moyen 
Duez : en prononçant ZZ fort \& dur comme ts, amolly ou mol \& blet comme vne nefle

en prononçant ZZ doucement comme dz I demy 2 milieu 3 moyen ${ }^{16}$

(s.v. MELE)

Oudin: miel. Prononcé e ouvert

prononcé e fermé, des pommes

Duez: I miel, en prononçant le premier E ouvert \& clair. 2 des pommes en prononçant le premier E fermé \& court.

Pour ce qui est du métalangage, Duez procède à une simplification en réduisant la fréquence des présentateurs métalinguistiques par rapport aux Recherches. Il utilise assez régulièrement, pour dire, pour, c'est à dire, c., alors que comme l'italien dit, nous dirions, cela se dit sont habituellement supprimés :

(s.v. aiguiser)

Oudin : s'aiguiser l'esprit, l'italien dit aguzzar i suoi ferruzzi

Duez : s'aiguiser l'esprit, aguzzare i suoi ferruzzi, aguzzarsi e assottigliarsi l'ingegno

Par contre, il maintient sans le modifier le système de marquage diatonique (mot vénitien, piedmontois, lombard, furlan, mot syrien), diatextuel (mot poëtique), diatechnique (terme de marine) et l'astérisque pour la variété diachronique, à l'exception du qualificateur en jargon car les entrées qui le comportaient chez Oudin sont exclues.

Plus généralement, les articles du Dittionario sont plus complets que ceux des Recherches. Duez enregistre souvent d'autres acceptions du même mot :

(s.v. *agghiadire)

Oudin : s'engourdir de froid ; sentir vn extréme froid

Duez : I s'engourdir \& se transir de froid, sentir vn extréme froid 2 auoir peur, estre saisi \& glacé de crainte

(s.v. gambòne)

Oudin : grosse jambe : gigot : jambon

Duez: I grosse jambe 2 gigot 3 jambon 4 tronc ou trognon et tige de chou ou de laituë \&c.

ou en donne une définition moins approximative et plus claire :

(s.v. MACARON)

Oudin : cosa fatta di pasta di mandole, zucchero acqua rosa \&c.

Duez : sortelletto o mostazzolo di marzapane fatto di pasta mandole zucchero acqua

rosa \&c. ${ }^{17}$

(s.v. ARROBA)

Oudin : une certaine mesure de vin ou d'huile, mot espagnol

Duez : un poids de 25 liures, \& une certaine mesure de vin ou d'huile, enuiron d'vn demy baril, mot espagnol

Parfois il glose :

(s.v. ARAZzo)

Oudin : tapisseries

Duez: tapis \& tapisserie figurée de diverses couleurs, appellée ainsi de la ville d'Arazzo en Perse, où il s'en fait beaucoup.

(s.v. ABELLINE)

Oudin : sorte d'auelines rouges au dedans

Duez : une sorte d'auelaines ou noisettes rouges au dedans appellées ainsi de la ville

Avellino en la campagne romaine dont est venu le mot auelaine

L'intervention de Duez se focalise surtout sur la phraséologie, qu'il accroît sensiblement ${ }^{18}$. Il recense des collocations, des locutions figées et des proverbes absents dans les Recherches : 
(s.v. ASNE) Le moulin est fermé les asnes se jouënt, il molino è serrato, gli asini trescano

(s.v PITEux) Vn piteux spectacle, un miserabile \& compassionevole spettacolo, un spettacolo orribile

Il est en piteux estat, egli è in meschino stato

Faire le piteux, fare il meschino \& il miserello

Il ajoute des acceptions et des traductions possibles :

(s.v. AGGIUNTA)

Oudin : Val più l'aggiunta che la carne, cela se dit quand vne seruante est plus belle que sa Maistresse

Duez: Val più l'aggiunta che la carne, cela se dit quand vne seruante est plus belle que sa Maistresse ; ou l'accessoire est meilleur que la chose mesme.

(s.v. POT)

Oudin : Dans un vieux Pot on fait de bonne souppes, l'Italien dit, gallina vecchia fà buon brodo

Duez : Dans un vieux pot on fait de bonne souppe, gallina vecchia fà buon brodo,

\& ad ogni stringa vecchia si posson metter puntali

Il étoffe les articles avec de nombreuses citations ou mots d'auteurs italiens :

(s.v. SOURDRE)

Oudin : sorgere, salire

Duez: sorgere, salire, escaturire Des fontaines qui sourdent des montagnes delle fontane che sorgono ò scaturiscono da'i monti. En l'essay des merveilles chap. 40

(s.v. GALONE,)

Oudin : costé

Duez : ${ }^{*}$ vn costé du corps. Ferì il gigante nel destro galone, il blessa le geant au costé droit, en Orlando le furieux

(s.v. ACCARNARE)

Oudin : acharner

Duez I acharner, ou gaigner \& prendre chair, comme fait vne playe en se guerissant 2 prendre par la chair, ou penetrer dans la chair auec les ongles ou auec autre chose 3 comprendre ou entendre

La piaga si accarna, la playe prend chair

Se ben l'intendimento tuo accarno, si je comprends bien ce que tu veux dire. Dante au 14. chant de purgatoire.

Grâce à la place de relief accordée à la phraséologie, Duez développe la dimension culturelle. Les articles se limitent de moins en moins au mot avec ses traduisants mais ils impliquent des définitions accompagnées de paraphrases, gloses, citations et références littéraires qui favorisent la découverte et la compréhension de la culture étrangère.

\section{Conclusion}

L'analyse du Guidon et du Dittionario révèle la manière dont Duez et la plupart des auteurs-professeurs de langues procèdent dans la réalisation de leurs ouvrages.

Ils n'hésitent pas à puiser dans des ressources déjà disponibles pour confectionner des produits, somme toute originaux, qu'ils peuvent exploiter à leur gré dans l'exercice de leur profession.

Il s'agit d'une opération de réélaboration intertextuelle délicate qui, dans le cas Duez, mériterait d'être étudiée plus amplement, en prenant en considération la totalité de 
son œuvre, pour tenter d'identifier un probable réseau de sources utilisé à plusieurs reprises.

C'est de cette opération qu'émergent ses qualités de grammairien, de lexicographe et surtout de pédagogue. Dans le Guidon, sa grammaire propose un parcours d'apprentissage 'à deux vitesses', qui met en avant les besoins des élèves, et les autres textes intègrent l'aspect pragmatique dans le processus d'acquisition de l'italien. Dans son Dittionario Duez fait une opération pédagogique remarquable qui consiste d'une part à organiser soigneusement le matériel lexicographique d'Oudin pour le rendre plus accessible et de l'autre à le perfectionner avec des informations plus étendues et moins imprécises.

Il est regrettable que Ferretti et les autres continuateurs des Recherches n'aient pas retenu les améliorations introduites par Duez.

BIBLIOGRAPHIE

\section{Éditions du Guidon...}

1641 Le Guidon de la Langve Italienne avec trois dialogues familiers, Italiens \& François. La comédie de la Moresse. Les complimens Italiens. Et une guirlande de proverbes. Leyde : Bonaventure et Abraham Elsevier, 286 p.

1650 seconde édition revue et corrigée par l'auteur, Leyde : Bonaventure et Abraham Elsevier, 270 p. ; 1659 Amsterdam : Louis \& Daniel Elzevier, 263 p.

1668 Amsterdam : Louis \& Daniel Elzevier, 263 p.

1670 Amsterdam : Daniel Elzevier, 256 p.

1673 Rouen : Guillaume de Luyne, 1673, 256 p.

1673 Rouen : Antoine Maurry, 1673, 256 p.

1684 Reveu, augmenté \& corrige, suivant les plus savans aucteurs tant vieux que modernes qui ont ecrit de cette langue. Avec trois dialogues familiers, italien \& francois. Lacomedie de la Moresse. Les complimens italiens. Une guirlande de proverbes. un traité de la poesie et à la fin les mots qui riment pour faciliter à faire les vers Une nomenclature copieuse italienne et françoise Un traitté des poincts et virgules. Plusieurs lettres. Avec les indices necessaires. Genève : Samuel de Tournes, $400 \mathrm{p}$.

1685 Genève : Samuel de Tournes, $400 \mathrm{p}$.

1685 Cologne : Bernard de Vangen, 400 p. ${ }^{19}$ 


\section{Éditions du Dittionario italiano e francese...}

1660-1659 Dittionario italiano e francese. Dictionaire italien et françois. Bien curieusement reueu, et augmente par Nathanel Duez. Premiere partie contenant les mots italiens expliqués en françois, Leyde : Jean Elsevier ; Seconde partie [...] contenant les mots françois expliqués en italien, Leyde : Jean Elsevier.

1662 Venise : Miloco

1664 Genève : Jean Antoine \& Samuel de Tournes

1664 Genève : Samuel Chouet

1671 Lyon : Antoine Beaujollin,

1671 Lyon : Michel Mayer

1671 Lyon : Claude Bourgeat

1671 Lyon : Jean Certe

1671 Lyon : Antoine Molin

1671 Lyon : Pierre Lecompagnon \& Robert Tallandier

1671 Lyon : Etienne Baritier

1671 Lyon : Pierre André

1678 Geneve : Samuel de Tournes

1678 Venise : Benedetto Miloco

\section{Sources primaires}

DUEZ, Nathanaël (1640). J. A. Comenii Janua aurea reserata quator linguarum, sive Compendiosa methodus latinam, germanicam, gallicam et italicam linguam perdidiscendi aucta, cum quadruplici indice, a Nathanaele Dhuez in idioma gallicum et italicum traducta. Leyde : Bonaventure \& Abraham Elsevier.

DUEZ, Nathanaël (1655). Esclaircissement de quelques differents en la langue Italienne. Leyde : François Hackes.

DULCIS Catharinus, (1599). Eunuchus Terentii iucunda metempsicoses in Aethiopissam mutatus. Comoedia lepidissima, in gratiam studiosorum linguae Italicae, opera \& studio Catharini Dulcis edita. Tübingen : G. Gruppenbach.

DULCIS, Catharinus (1605). Schola italica, in qua praecepta bene loquendi facili methodo proponuntur. Francfort : Wolfgang Richter \& Konrad Neben. Autres éditions : 1614 Franfort : Peter Maus ; 1616 Francfort : Peter Maus ; 1620 Cologne : Peter Henning ; 1631 Cologne : Peter Henning ; 1641 .

FERRETTI, Laurent (1662-1663). Dictionnaire italien et françois. Contenant les recherches de tous les mots italiens expliquez en françois, auec plusieurs proverbes \& phrases, pour l'intelligence de l'vne \& l'autre langue Par Antoine Oudin, Secretaire Interprete du Roy. Revu, corrigé \& augmenté, non seulement d'une quantité de mots mais aussi d'un grand nombre de Phrases, proverbes \& Locutions necessaires, auec les accents pour prononcer les syllabes longues ou 
breves \& les irregulatitez \& Annomalies des verbes Par Lavrent Ferretti. Paris : Antoine de Sommaville.

oudiN, Antoine (1640). Recherches italiennes et françoises ou dictionnaire contenant outre les mots ordinaires, une quantité de proverbes \& de phrases, pour l'intelligence de l'une \& l'autre langue. Paris : Antoine de Sommaville. Autres éditions : 1643 Paris : Sommaville ; 1653 Paris : Sommaville; 1655 Paris : Sommaville.

\section{Sources secondaires}

BINGEN, Nicole (1987). Le maître italien. Bruxelles : Émile Van Balberghe.

DARDI, Andrea (1992). Dalla provincia all'Europa, L' influsso del francese sull'italiano tra il 1650 e il 1715.

Florence : Le Lettere.

DE BOER, Minne G. (1996). « Pieter Staat, de Vrouwen Spinnen. Naar aanleiding van de Heruitgave van Lodewijk Meyers Italiaansche Spraakkonst, 1672 ». Incontri. Rivista europea di Studi Italiani, 11, 65-82. En ligne : <http://webdoc.ubn.kun.nl/tijd/i/incontri/> (15 janvier 2016).

DODDE, Nantko Lieven (1997). « Franse scholen van 1482 tot 1857 ». Meesterwerk 9, 2-7.

JONES, William Jervis (2000). German Lexicography in the European Context. A descriptive bibliography of printed dictionaries and word lists containing German language (1600-1700). Berlin/New York.

LILLO, Jacqueline (éd.) (2008). 1583-2000 : Quattro secoli di lessicografia italo-francese. Repertorio analitico di dizionari bilingue. Berne : Peter Lang.

LILLO, Jacqueline (éd.) (2013). Les best-sellers de la lexicographie franco-italienne. XVI ${ }^{e}-X X I^{e}$ siècle. Rome : Carocci.

Lo CASCIO, Vincenzo (1997). « L'italiano nel secolo d'oro olandese ». In Harro Stammerjohann (éd.). Italiano lingua di cultura europea. Atti del simposio internazionale in memoria di Gianfranco Folena, Weimar 11-13 aprile 1996. Tübingen : G. Narr, 247-281.

LOONEN, Pieter L. M. (1993). « Nathanael Duez as an example of a distinguished language master in the seventeenth century ». In Jan Noordegraaf \& Frank Vonk (eds.). Five hundred Years of Foreign Language Teaching in the Netherlands 1450-1950. Amsterdam: Stichting Neerlandistiek, 57-66.

LOONEN, Pieter L. M. (1994). « The influence of Comenius on modern language teaching ». Paradigm 15. En ligne :

http://faculty.education.illinois.edu/westbury/paradigm/loonan.html> (15 janvier 2016). LOONEN, Pieter L. M. (1995). « Nathanael Duez. Biography and a first bibliography », Meesterwerk. Berichten van het Peeter Heynsgenootschap, 3, 2-15.

LOONEN, Pieter L. M. (2000). « The influence of the Huguenots on the Teaching of French in the Dutch Republic during the $17^{\text {th }}$ century ». In Jan De Clercq, Nico Lioce \& Pierre Swiggers (éds). Grammaire et enseignement du français. 1500-1700. Louvain : Peeters, 317-333.

MATTARUCCO, Giada (2003). Prime grammatiche d'italiano per francesi (secoli XVI-XVII). Florence : Accademia della Crusca.

MINERVA, Nadia (1991). « Jean Vigneron dit Veneroni (1642-1708)» La Lettre de la SIHFLES 11, 8-10. MINERVA, Nadia (2007). « Représentations de l'autre. L'italien et les italiens dans quelques dictionnaires bilingues des XVII ${ }^{\mathrm{e}}$-XVIII ${ }^{\mathrm{e}}$ siècles) ». In Kibbee Douglas A. (éd) History of linguistics 
2005 , selected papers from the 10th International conference on the History of the Language Sciences (ICHOLS X), 1-5 september 2005. Amsterdam/Philadelphie : John Benjamins, 308-320.

MINERVA, Nadia (2013). « Un siècle de lexicographie bilingue : le Dictionaire de Giovanni Veneroni et ses adaptations ». In Lillo, Jacqueline (éd.) (2013). Les best-sellers de la lexicographie francoitalienne. $\mathrm{XVI}^{e}-\mathrm{XXI}^{e}$ siècle. Rome : Carocci, 33-51.

MORMILE, Mario (1989). L'italiano in Francia e il francese in Italia : storia critica delle opere grammaticali francesi in Italia ed italiane in Francia dal Rinascimento al primo Ottocento. Turin : Albert Meynier. MORMILE, Mario (1993). Storia dei dizionari bilingui italo-francesi. La lessicografia italo-francese dalle origini al 1900. Fasano : Schena.

MURANO, Michela (2012). Des phrases aux séquences figées. La phraséolgie dans les dictionnaires bilingues franco-italiens (1584-1900). Bologne : Clueb, Quaderni del Cirsil 11.

PFISTER, Max (1989). L'importance d'Antoine Oudin pour la lexicographie française et italienne. In Marcella Giacomelli-Deslex et al. La lingua francese del Seicento. Bari-Paris : Adriatica-Nizet, 91-103. Szoc, Sara (2009). « Esclaircissement sur deux maîtres plurilingues du XVII ${ }^{\mathrm{e}}$ siècle à Leyde ». Documents pour l'histoire du français langue étrangère ou seconde 42, 65-86. En ligne :

<http://dhfles.revues.org/692> (15 janvier 2016).

VAN PASSEN, Anne-Marie (1981). « Appunti sui dizionari italo-francesi apparsi prima della fine del Settecento ». Studi di lessicografia italiana 3, 29-65.

VAN STRIEN-CHARDONNEAU, Madeleine \& KOK ESCALLE, Marie-Christine (2010). « Le français aux PaysBas (XVII -XIX siècles) : de la langue du bilinguisme élitaire à une langue du plurilinguisme d'éducation ». Documents pour l'histoire du français langue étrangère ou seconde 45, 123-156. En ligne : <http://dhfles.revues.org/2448> (15 janvier 2016). VANVOLSEM, Serge (2000). « The sources of the first Italian grammar in Dutch », In Piet Desmet et al. (eds.). The history of linguistic and grammatical praxis. Louvain: Peeters, 97-112.

\section{NOTES}

1. Pour une bibliographie complète $c f$. Loonen (1995) et Jones (2000: 298-328).

2. Il publia aussi l'Esclaircissement de quelque differents en la langue Italienne (Duez 1655), un recueil de remarques sur la langue italienne occasionnées par une querelle qu'il eut avec un autre maître de langue, Pietro Paravicino. Cf. Szoc (2009) qui prend en considération également le Guidon.

3. Ils enseignaient aux adultes mais aussi dans le réseau d'écoles françaises qu'ils avaient fondées pour former les jeunes (Dodde 1997).

4. À partir de 1684 les éditions comportent aussi un Traitté comprenant une bréve instruction de la poesie italienne (qui n'est autre que la troisième partie de la Nouvelle méthode pour apprendre facilement en peu de temps la langue italienne de Claude Lancelot, Paris : P. Le Petit, 1660), un Rimario delle voci della lingua italiana per facilitare à compor versi, une nomenclature intitulée Nouveau vocabulaire italien françois contenant les mots qui tombent le plus souvent dans l'entretien ordinaire - Dizionario delle cose che s'usano il più ne' discorsi famigliari, un Trattato del modo di puntare, des Lettere italiane.

5. Il s'agit d'un manuel d'italien. Catherin Ledoux ou Catharinus Dulcis (1540-1626), philologue originaire de la Savoie, de foi protestante, enseigna l'italien et le français à Cassel et Marbourg. Sur lui $c f .<h t t p: / / w w w . d u l c i s-i n f o . d e / v i t a . h t m l>$. Il aurait puisé la matière des dialogues dans les Second frutes de John Florio (Mattarucco 2003 : 29). 
6. Meyer (1629-1681), philosophe du cercle de Spinoza, étudia la médecine et le latin à l'Université de Leyde.

7. Per visitare alcuno nuovamente arrivato di viaggio, per offerirsi a servire, per querelarsi con chi non ha voluto ricever qualche offerta, per rallegrarsi della salute di alcuno. per rallegrarsi con alcuno di dignità ottenuta, per rallegrarsi con altri di heredità acquistata. per augurar sanità. per augurar dignità, per augurar prosperità che servirà anco per augurio delle buone feste, per iscusarsi di non haver potuto scrivere, per iscusarsi d'esser troppo importuno, per iscusarsi di negligenza apparente, per lodar gli scritti di alcuno, per condolersi della morte di qualcheduno, per condolersi di qualche travaglio, per domandar qualche cortesia, per domandare consiglio, per ringratiar d'una cortesia o di qualche favore, per donare, \& ringratiar di dono ricevuto, per invitare a desinare, per invitare a nozze, per invitare ad esser compadre, per raccomandare, per pigliar licenza di alcuno, per offerirsi nella partenza.

8. Les deux premières éditions de 1641 et de 1650 ne contiennent pas la section intitulée $D u$ mouvement des noms, tant adjectifs que substantifs, qui est consacrée à la formation du féminin et à l'accord du participe passé.

9. À ce sujet, dans l'édition de 1685 , le libraire justifie encore plus clairement la fonction des différents textes. Pour les dialogues: "E' cosa incredibile di quanto aiuto siano ad uno che comincia ad imparare una lingua, simil sorte di dialoghi, sendo che oltre che servono à far capire bene la formazione de' periodi. Danno altresì un'idea perfetta del modo del parlare usuale e familiare della nazione, che si serve di tal lingua ». La Comédie est utile " perché vi sono contenute certe espressioni burlesche che ti potranno dar cognizione dello stile triviale degl'Italiani, si come il rimanente te la dà dello stile maestoso, e civile, affinchè si possa sapere tutto quello, che può occorrere in una lingua ». Les Complimenti servent car "Gl'Italiani hanno certi complimenti più gravi ed ampij delle altre nazioni ». La Ghirlanda «farà conoscere la forza, e l'uso de' proverbi ».

10. Pour une description détaillée nous renvoyons à Lillo (2008: fiches $32,33,35,36,37,38,39$, 40, 41, 43, 44), Bingen (1987: 77-84) et Van Passen (1981). Nous avons également consulté les catalogues en ligne des principales bibliothèques européennes et américaines pour vérification. Toutes les éditions comportent le même nombre de pages ( 970 pour la première partie et 605 pour la deuxième) et la même disposition du texte sur deux colonnes sauf les deux éditions vénitiennes qui présentent trois colonnes et donc un nombre réduit de pages (452 et 288).

11. Lorenzo Franciosini, (1600-1645), toscan, hispaniste, grammairien et traducteur, avait publié le Vocabolario italiano, e spagnolo (Rome : G. Angelo Ruffinelli \& Angelo Manni,1620).

12. Cette étude ne s'était pas attardée sur le Dittionario.

13. Duez en ajoute certains, comme « ALEP, HALEPPE, vne ville de Surie », ou « LUNIGIANA, LUNIGEANE, nom d'vne ville jadis sou les Genevois [sic] ». Chez Oudin il n'y avait que le dicton « Chi non fà la lunigiana è figliuol di puttana » que Duez maintient.

14. À titre d'exemple, voici quelques entrées des Recherches supprimées : «MAGRA, en jargon, la Mort »; « MARIANO, en jargon miracle »; « MARTINA en jargon espée ».

15. L'entrée CONZALAVEZZI (ou CONCIALAVEZZI) est d'ailleurs absente.

16. Ferretti semble avoir intégré les précisions du Dittionario :

MEZzo : prononcé rude, passé, sec, flestry, pron. l'e fermé, \& les zz comme ts.

MEZzo demy : mileu : moyen : pron. l'e ouvert \& les zz comme dz.

17. L'article Mostacciuoli est présent dans les deux dictionnaires.

18. Son attention est confirmée par cette affirmation de la Préface : «Et là où il $\mathrm{y}$ a quelques phrases ajoustées à vn mot c'est pour monstrer vne particulière construction et vn usage remarquable de telle parole ou pour la dignité de quelque façon de parler fort notable». Le traitement de la phraséologie dans le Dittionairo a été étudié par Murano (2012 : 48-53). 
19. Cette édition, identique à l'édition de 1684 et 1685 , n'est indiquée dans aucun des répertoires bibliographiques disponibles. De repérage difficile, nous l'avons consultée à la Bibliothèque de San Salvatore Monferrato (cote : FVA.1209).

\section{RÉSUMÉS}

Nathanaël Duez, enseignant d'italien, de français et d'allemand, est l'auteur de manuels de langue et de dictionnaires qui eurent un succès remarquable au XVIIe siècle. Nous nous intéressons à sa production pédagogique concernant le domaine linguistique italien qui comprend une grammaire, Le Guidon de la langue italienne, et le Dittionario Francese Italiano, en les situant dans le cadre des activités des professeurs de langues opérant aux Pays-Bas. Nous en donnons une description. L'analyse comparée du Ditionnario et des Recherches italiennes et françoises d'Antoine Oudin montre que Duez se place dans le sillage d'Oudin tout en innovant et met en évidence les points de force du Dittionario. Les modifications qu'il apporte, répondent aux exigences dictées par sa fonction d'enseignant et vont dans la direction d'une simplification de la structure des articles et d'un enrichissement des contenus.

A teacher of Italian, French, and German, N. Duez authored language textbooks and dictionaries that obtained a great success in the $17^{\text {th }}$ century. My focus is on his educational production concerning the area of Italian, which includes a grammar (Guidon de la Langue italienne) and a dictionary (Dittionario Francese Italiano). They belong to the context of language teachers working during that period in the Netherlands and I provide an illustration of the background. My comparative analysis of the Dittionario and Antoine Oudin's Recherches intends to show that Duez actually follows, but also updates, Oudin's models, and I also highlight the marked qualities of the Dittionario. Duez's changes are justified by the nature of his professional role as a teacher, and aim on the one hand to simplify the structure of the entries, and on the other to expand their contents.

\section{INDEX}

Mots-clés : Duez, enseignement du français et de l'italien, grammaire italienne, Pays Bas au XVIIe siècle, lexicographie franco-italienne

Keywords : Duez, teaching of French and Italian, Italian grammar, 17th century's Low Countries, franco-italian lexicography

\section{AUTEUR}

\section{ANTONELLA AMATUZZI}

Université de Turinantonella.amatuzzi@unito.it 